Recepción: 20 / 04 / 2017

Aceptación: 20 / 05 / 2017

Publicación: 15 / 09 / 2017

Ciencias Sociales y políticas

Artículo Científico

\title{
Influencia de la Publicidad exterior en los hábitos alimenticios de los estudiantes de la Universidad de Guayaquil
}

Influence of outdoor advertising in the eating habits of students of the Universityof Guayaquil

\section{Influência da publicidade ao ar livre nos hábitos alimentares de estudantes da Universidade de Guayaquil}

Lcda. Luci Cristina Salas Narváez ${ }^{\text {I }}$

luci.salasn@ug.edu.ec

Sr. Daniel Andrés Angulo Vélez II

daniel.angulovel@gmail.com

Sr. Daniel Fernando Díaz Tandazo III

danielfernando1981@hotmail.com

\section{Correspondencia: luci.salasn@ug.edu.ec}

I. Magíster en Administración de Empresas con mención en Marketing y Recursos Humanos, Licenciada en Ciencias de la Educación especialización en Mercadotecnia y Publicidad, Docente de la Universidad de Guayaquil, Guayaquil, Ecuador.

II. Ingeniero en Marketing y Negociación Comercial de la Facultad de Ciencias Administrativas de la Universidad de Guayaquil, Ecuador.

III. Ingeniero en Marketing y Negociación Comercial de la Facultad de Ciencias Administrativas en la Universidad de Guayaquil, Guayaquil, Ecuador. 


\section{Resumen}

La presente investigación tiene como objetivo principal, el análisis sobre la influencia que genera la publicidad exterior hacia los hábitos alimenticos o conductas alimenticias que se mantiene durante la vida estudiantil; así mismo, como el mensaje publicitario logra interceder al consumo de alimentos poco saludables; pues se cree que la publicidad exterior visualizada en sectores cercanos a la Universidad de Guayaquil, intercede e influye negativamente en lo que consumen diariamente los estudiantes. Aplicando una metodología de investigación cuantitativa como cualitativa, que ayude a valorar el número aproximado de estudiantes influenciados por la publicidad externa, que se visualiza en los locales comerciales alrededores de la Universidad de Guayaquil. Las técnicas y herramientas están basadas por encuestas, observación, y grupos focales que justifiquen la investigación realizada. Los resultados del estudio permitirán el diseño de un modelo de comunicación publicitaria, con la finalidad de que se pueda minimizar considerablemente el consumo masivo de productos alimentarios no saludables fomentando una alimentación sana que desarrolle mejor el desempeño estudiantil.

Palabras claves: Influencia publicitaria, conductas alimenticias, nutrición, estudiantes, Universidad de Guayaquil 


\begin{abstract}
The main objective of the present investigation is the analysis of the influence of outdoor advertising on the eating habits or eating habits that are maintained during the student life; Likewise, as the advertising message manages to intercede with the consumption of unhealthy foods; As it is believed that advertising is displayed in the following sectors near the University of Guayaquil, it intercedes and negatively influences what students consume daily. Apply a qualitative quantitative research methodology to help evaluate the approximate number of students influenced by external advertising, which is displayed in the commercial premises of the University of Guayaquil. The techniques and tools are based on surveys, observation and focus groups that justify the research done. The results of the study allow the design of a model of public communication, in order to considerably minimize the mass consumption of unhealthy food products to promote healthy eating that develops the best student performance.
\end{abstract}

Key words: Influence of advertising, eating behavior, nutrition, students, University of Guayaquil 


\section{Introducción.}

Durante el tiempo de estudiantes, es importante seguir requerimientos básicos de nuestro organismo, como es la alimentación. Por tal motivo es fundamental que durante los años de estudio esta sea saludable. Pero las conductas alimenticias adquiridas por los estudiantes en la actualidad, son de productos con un escaso valor nutricional haciéndoles padecer a largo plazo problemas tales como la obesidad, hipertensión arterial, diabetes tipo 2 que son las principales enfermedades y causa de muerte en el Ecuador. Cabe indicar que el índice de sobrepeso en el Ecuador según el Ministerio de Salud (2015) ha ido en aumento y siguen en ascenso (especialmente en adolescentes en edad escolar con un $37,2 \%$ ), debido al mal manejo en las conductas alimenticias, ya que se está expuesto a diversos factores e inconvenientes y aunque la adopción de un estilo de vida saludable a través de la alimentación, el entorno no es el mejor aliado a la hora de consumir un alimento.

En muchas ciudades del mundo "la publicidad exterior forma parte del escenario urbano. Ciudades de todos los continentes tienen publicidad exterior que participa de su cotidianeidad con mensajes llamativos y atractivos, e incluso divertidos." (Breva \& Mut, 2016) Se considera que la publicidad influye mucho en los comportamientos fisiológicos del consumidor, al persuadidor a la toma de decisión con referente a los alimentos sin importar lo cuan saludables sean estos. Ya sea en este caso como los lugares públicos, esta logra desarrollarse por medio de carteles, rótulos, todos aquellos soportes que se instalan en lugares de exposición pública o donde se desarrollan espectáculos, eventos culturales, en que se pueda llegar a la mente del consumidor.

Es por ello que el estudiante de la Universidad de Guayaquil entra como objeto de estudio, ya que debido a la agenda en sus actividades diarias, logra ser influenciado por el contenido publicitario que visualiza en los exteriores de un local comercial a la hora de alimentarse, y este a su 
vez lo motiva al consumo de alimentos perjudiciales para la salud. Por lo que, a través de este estudio se pretende contribuir al mejoramiento de los conductas alimenticias en los estudiantes de la Universidad de Guayaquil.

\section{Marco teorico}

\section{La Publicidad exterior}

Para tener claridad sobre el tema, "La publicidad, comunica beneficios o contenidos de imagen de productos, marcas y servicios o empresas teniendo como objetivo principal vender" (Rosa, 2010). Entre los más importantes tipos de publicidad, se encuentra la publicidad externa o exterior que "se encuentra formada por carteles, afiches, rótulos luminosos y aquellos soportes que se instalan en lugares públicos o en la fachada de un establecimiento comercial como parte de un comercio" (Erizagaba, 2013)."

O a su vez es “aquella comunicación al aire libre a través de vallas o carteles, pero su contenido se limita a una imagen y un mensaje no más de ocho palabras" (Guinn, Allen, \& Semenik, 2010). Aunque es una publicidad de corta duración, el contenido del mensaje debe tener un impacto óptico atrayente y debido a la limitación de tiempo para visualizarlo debe reducir en sus formatos los números de elementos que conforman el anuncio. Ya que, "La colocación de avisos y mensajes persuasivos, en tiempo o espacio, comprado en cualesquiera de los medios de comunicación intentan informar y/o persuadir a un mercado meta en particular o a audiencias acerca de sus productos, servicios" (American Marketing Asociation, 2005)

Pero se debe identificar, que mucha de las publicidades que se exhiben fuera de un local comercial, no está acorde a la venta de una imagen de marca, ya que resulta propio de alguien cuyo 
único conocimiento de la publicidad procede a la intención de crear un mensaje con el fin de obtener una rentabilidad, por lo tanto esto no es hacer publicidad, sino "anunciar" para tener notoriedad de lo que se ofrece a la venta. Ya que la publicidad no solo ofrece el anuncio de mercancía, sino a su vez una imagen del producto.

\section{El estudiante universitario y la nutrición}

El estudiante en esta fase actúa por sus propias decisiones; en donde seguir una nutrición adecuada le permite mantener la integridad funcional y estructural de las neuronas. Y en general durante los días de estudios, se recomienda una dieta variada y equilibrada; basándose en el consumo moderado de todo tipo de alimentos.

Pero en muchos de los casos, el estudiante universitario no logra cumplir con estos requisitos, sobre llevar una alimentación saludable, por lo que se generan trastornos alimenticios, que a largo plazo acarrearán problemas de salud. (Ochoa \& Ojeda, 2013) Cabe recalcar que este es un fenómeno social que empieza desde casa o, en muchos casos debido a la mala influencia de los alrededores por las costumbres adquiridas de satisfacer una necesidad vital de alguna manera.

Los nutricionistas no solo deberán anunciar las recomendaciones actuales, sino que también deben justificar teniendo en cuenta que "el patrón alimenticio de este grupo se caracteriza por el alto consumo de dulces, golosinas, vegetales $\mathrm{C}$, cereales y aumento en la preparación de frituras; al mismo tiempo que es bajo el consumo de lácteo, verduras y frutas.” (Rios \& Mateos, 2009)

“Los comportamientos personales de salud están determinados por una amplia variedad de factores, incluyendo influencias socioculturales" (Salas, 2011). Por lo tanto, las conductas de salud personal son medidas por una amplia variedad de factores que incluyen por supuesto la influencia 
cultural y social en los estudiantes; en estos casos no cumplen con estos requisitos para llevar a cabo una alimentación saludable, dejándose influir a una conducta alimenticia que visualmente se ve saludable.

"Y es que el consumo excesivo de café, tabaco o comida "chatarra", inevitablemente le hace mal al cuerpo, contrario a que se realice actividad física regular, la evitación de la grasa en la dieta, el comer fruta y la fibra" (Hugalde, 2013), ayudaran a que las conductas alimenticias pueden ser positivas en el estudiante a través de actividades que ayuden a prevenir enfermedades como la obesidad, enfermedades cardiacas a temprana edad, es un hecho que comer frutas y cuidar la salud a través de actividad física forman parte de una conducta de salud positiva.

\section{La publicidad externa como influencia en hábitos alimenticios}

"La influencia es un proceso social en virtud del cual un individuo o grupo logra que otro individuo o grupo actúe bajo las expectativas del otro". (Buchelli, 2008). Mientras que "La conducta alimenticia se define como el comportamiento normal relacionado con: los hábitos de alimentación, la selección de alimentos que se ingieren, las preparaciones culinarias y las cantidades ingeridas de ellos". (Osorio, 2012). Por lo tanto, el ser humano influye su conducta alimenticia con preferencias o rechazos a los alimentos que generan emociones sensoriales (sabor, olor, textura) o por la influencia visual (color, texto).

Desde el punto de vista nutricional, la alimentación humana está fuertemente vinculada al aspecto fisiológico, debido al aporte de ciertos componentes y nutrientes que favorecen o limitan (tales como: proteínas, sodio, fibra, almidón, azúcar, alergenos potenciales, etcétera), a su textura, a la manera como son preparados para su ingestión y a la posibilidad de ser digeridos y asimilados por el organismo humano (Casanueva, Kaufer-Horwitz et al, 2001). Por otra parte se puede indicar que 
"la publicidad afecta directamente a la salud y a la nutrición de las personas debido a que incentiva a comprar y consumir alimentos pocos saludables con el único fin que solo es vender”. (Todo Marketing, 2014).

Y es que para el punto de vista nutricional, las campañas publicitarias de alimentos y bebidas no contribuyen a una sana alimentación; marcas como Mc Donalds, Burger King, Coca-cola o Pepsi solo influyen en los jóvenes a consumir estos productos que poseen un poco valor nutricional. Ya que, "la influencia de la publicidad en el consumidor será negativa o positiva según el uso que se haga de ella" (Fischer \& Espejo, 2013). Esto no quiere decir que exista publicidad noble, sin embargo se debe tener mucho cuidado al utilizar un mensaje para captar la atención del público objetivo o para persuadirlo más eficazmente, sin inducir en el error de afectar al determinado comportamiento que afecta seriamente la imagen de la empresa.

Ya que la publicidad siempre influenciara de alguna manera al consumidor ya que es el objetivo inicial, pero al final es decisión de la audiencia el consumir este tipo de alimentos poco saludables.

\section{Metodología.}

Para la valoración de datos la metodología empleada consta de la encuesta como técnica cuantitativa, y a su vez el método de observación como técnica cualitativa misma que consta en la observación del comportamiento alimenticio de los estudiantes; además de ello observar los locales comerciales frecuentados por el estudiante.

Dentro de la técnica cualitativa se justificará con método grupo focal, mismo que constará de una reunión entre los estudiantes de la universidad con el fin de conocer que influye al 
comportamiento de compra de alimentos pocos saludables. Por otro lado, la población o las unidades de análisis que fueron parte del objeto de estudio, y la muestra de este cálculo, que fueron con una población de 52.148 estudiantes de la Universidad de Guayaquil, tomando una muestra aleatoria simple estratificada de 400 estudiantes en toda la Universidad.

Los datos que se recopilaron en esta investigación de manera cuantitativa, fueron procesados con el fin de establecer una base de datos de información y posteriormente fueron analizados e interpretados de manera obtener una conclusión de la investigación.

\section{Resultados.}

Dentro de la investigación, los resultados indican que los estudiantes son consientes que dentro de la dieta alimenticia alimentos como carnes, huevos, leche (38\%) son una importante fuente de nutrición para el desarrollo diario de sus actividades, al igual que el consumo de frutas (27\%), pues estas aportan las vitaminas necesarias al organismo. Por otra parte el consumo de vegetales y frutas durante las horas de actividades académicas es poco frecuente $(27,5 \%)$.

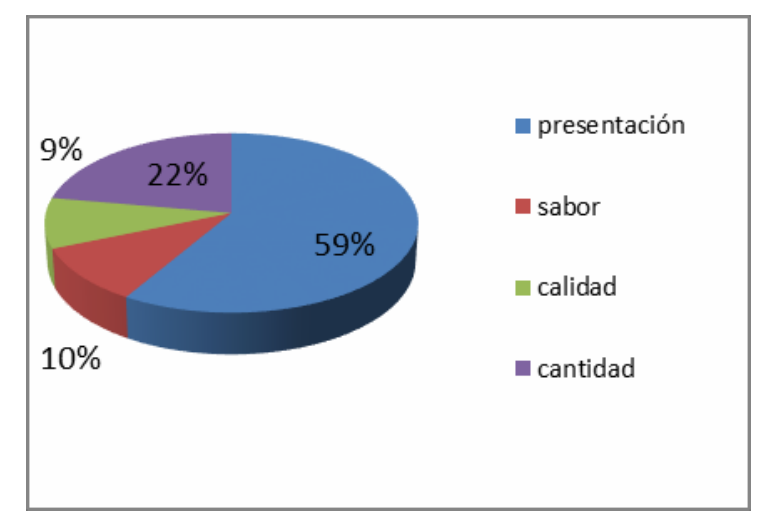

Figura 1. Alimentos que deben consumir en la dieta alimenticia. 


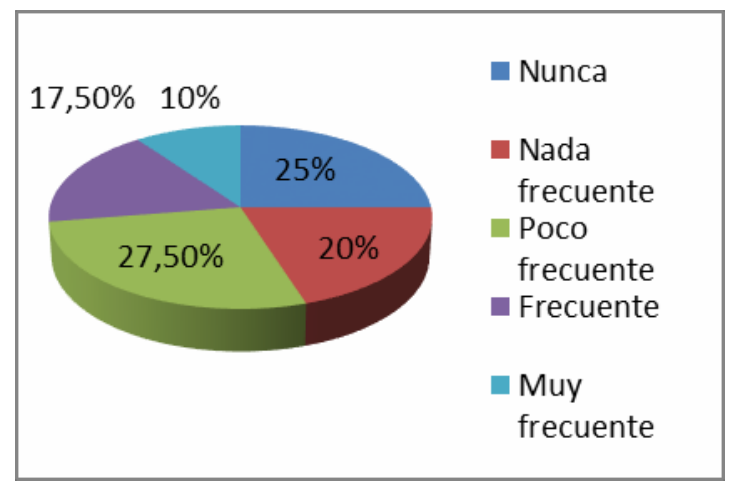

Figura 2. Frecuencia de consumo de frutas, vegetales en jornada estudiantil.

Los datos indican, que los estudiantes no se están alimentando sanamente $(70 \%)$, lo que puede ser perjudicial en la salud del estudiante. Ya que se dejan influenciar por los diferentes tipos de publicidad exterior que son llamativas de ámbito visual como económico (73\%) mismas que encuentran en los alrededores de la Universidad de Guayaquil, por lo cual, existe una dependencia altamente significativa entre las variables publicidad exterior y hábitos alimenticios.

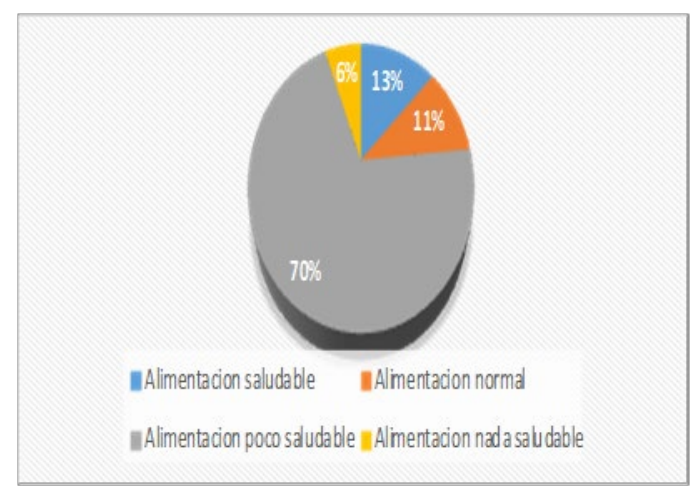

Figura 3 Tipo de alimentación de la población universitaria

Tomado de: Encuestas realizadas a los estudiantes de la universidad de Guayaquil. (2015) 


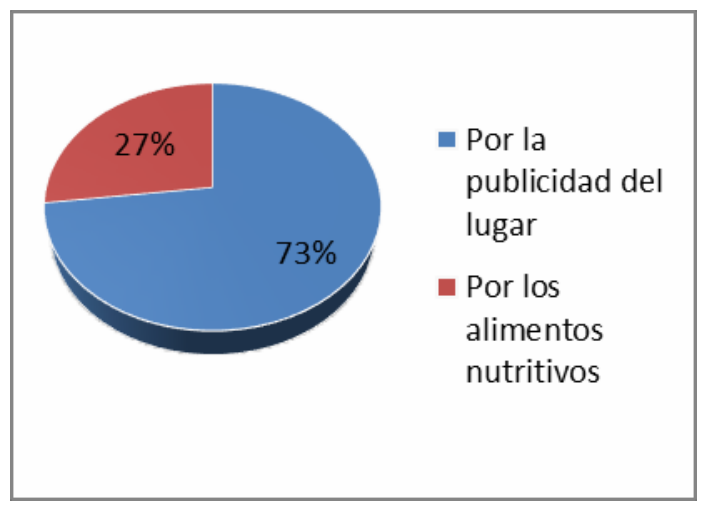

Figura 4. Al momento de elegir un alimento, lo consume por

\section{Tomado de: Encuestas realizadas a los estudiantes de la universidad de Guayaquil.}

Cabe indicar que los encuestados, creen que un punto importante a la hora de adquirir/consumir alimentos en restaurantes cercanos a la Universidad de Guayaquil, si bien, se dejan influenciar por el mensaje publicitario, también comprueban que la presentación del producto (59\%) sea como lo refleja en la imagen del anuncio a su vez que contenga buena cantidad (22\%) del producto que consumirán. A su vez, los estudiantes consideran que debe haber un establecimiento dentro de las instalaciones universitarias con comida saludable, de esa manera ellos consumirían una dieta balanceada. Sin recurriría a la conocida "comida chatarra" que se venda fuera.

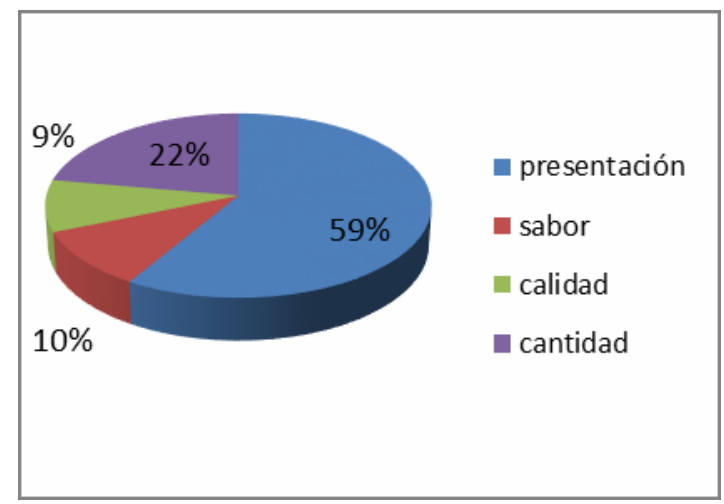

Figura 5. Características considera importantes a la hora de comprar comida en restaurantes. 


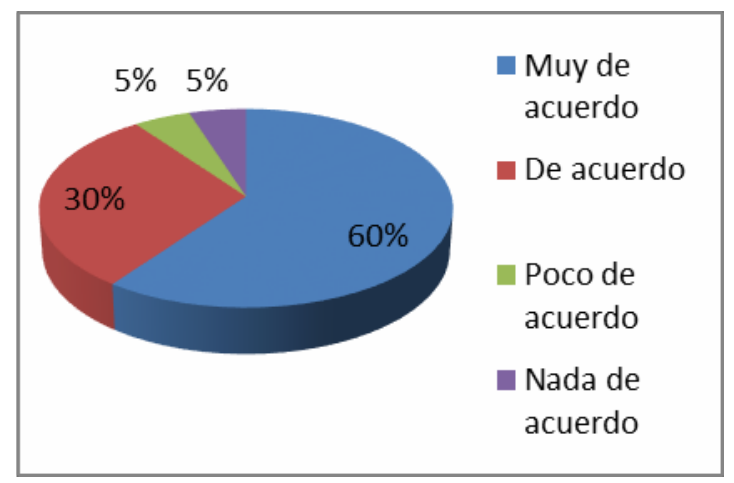

Figura 6. Dentro de la Universidad de Guayaquil, debería haber restaurantes y lugares de comida saludable.

Dentro del grupo focal realizado, se pudo evidenciar en cuanto a las respuestas dadas por los ocho participantes (entre ellos hombres y mujeres elegidos de manera aleatoria). Recalcaron tener conocimientos claros sobre una conducta alimenticia saludable en la sociedad universitaria, pero por el contrario son cada vez menos apreciables, algunos participantes acotaron que no disponen del tiempo necesario para almorzar en sus hogares debido a sus actividades que los obliga a permanecer en la Universidad casi durante todo el día.

A su vez, coinciden en que diariamente compran/consumen alimentos poco saludables como pasteles, sándwiches o tostadas, otros tres panelistas aseguran que no son alimentos totalmente nutritivos pero que aun así son deliciosos como económicos, mientras otros también indican que por motivos de tiempo y necesidad de llenar sus estómagos deben consumirlos pese a que el costo de estos en algunos casos es muy elevado.

Cabe indicar, que los integrantes concuerdan en que la publicidad que visualizan en los alrededores de la universidad de Guayaquil es muy directa y que de alguna forma influye a su conducta alimenticia. 
También se les presentó a los miembros del equipo focal diferentes afiches sobre productos nutritivos (ensaladas, frutas y vegetales) a lo cual, respondieron que las frutas si se comercializan en algunos locales cercanos a la Universidad, sin embargo sus costos son realmente altos y es poca la cantidad ofrecida. Al apreciar estos afiches consideran que se está contribuyendo al cambio de una conducta alimenticia, donde no se apoyan el consumo de alimentos altos en grasas y con bajo aporte nutricional que es importante para el desarrollo estudiantil.

Como aporte final, una vez analizadas las percepciones y comentarios del grupo focal, se llegó a la conclusión de que los alumnos en más de una ocasión se han sentido persuadidos al visualizar el contenido publicitario de un local comercial sin considerar si el alimento es o no saludable, y que no contribuye a mantener una conducta alimenticia balanceada; sin embargo también se pudo concluir que gran parte de los estudiantes están conscientes de cómo influye este tipo de publicidad ante los alimentos que consumen, ya que consideran a la publicidad como parte de su vida y no la perciben activamente como influencia.

\section{Discusión}

Se consideró a través de los datos encuestados, que la publicidad en locales comerciales cercanos de la Universidad de Guayaquil, si influye al momento de la toma de decisión al consumo de alimentos; los estudiantes consideran que la comida que ofrecen los restaurantes es de tipo normal para el consumo, dado que cumplen con las expectativas de los estudiantes.

Se debe considerar que la poca disponibilidad de tiempo, también influye en que los estudiantes no puedan mantener una dieta balanceada y elijan consumir alimentos rápidos para reintegrarse a sus actividades. El justificante para su consumo son las altas jornadas de estudio que poseen los estudiantes en su agenda académica, por lo cual, el estudiante opta por alimentarse con 
comida rápida para estar en condiciones aptas para sus clases, sin considerar si es perjudicial al futuro.

Otro punto es el factor económico, ya que algunos estudiantes no cuentan con una economía alta para comprar/consumir alimentos saludables, y en su efecto se dejan influir por la publicidad, en donde se ofrece precios accesibles por consumir alimentos de comida rápida. A su vez se concluye que el proporcionar de un lugar fresco y amplio con productos nutritivos y económicos por parte de la institución seria una alternativa para la mejora alimenticia. Un espacio que incluya anuncios publicitarios con productos nutritivos y precios módicos que beneficie a los estudiantes a encontrar una nueva variante alimenticia, ya que actualmente los universitarios están inmersos en una publicidad engañosa por parte de algunos locales comerciales en los alrededores de la Universidad de Guayaquil.

\section{Conclusion}

Este estudio concluye que la publicidad si desempeña un papel importante e influye en lo que consumen los estudiantes. Y es que de cierta manera se promueve inconscientemente el consumo de alimentos altos en grasas, azucares y carbohidratos que generan una conducta alimenticia poco saludable. La Organización Mundial de la Salud promueve que las campañas publicitarias o anuncios sean mas explícitos en los ingredientes que contienen los alimentos que se venden. Con el fin de erradicar el índice de obesidad como de enfermedades cardiovasculares que en jóvenes cada vez mas están presentes.

Cabe indicar que la forma de alimentarse de los estudiantes de la universidad de Guayaquil, carece de conciencia nutricional, y esta se debe a que están siendo influenciados por la publicidad exterior que presentan locales comerciales cercanos a la Universidad de Guayaquil, lo que ha 
desarrollado una ineficiente cultura alimentaria que a la vez influencia a los compañeros, amigos e inclusive podría arraigarse hasta los hogares de los Universitarios.

Por lo que es importante utilizar la publicidad de manera positiva con el fin de promover una dieta sana y equilibrada, excluyendo este tipo de alimentos pocos saludables.

\section{Bibliografia.}

Breva, E., \& Mut, M. (2016). El desarrollo de las ciudades desde la publicidad exterior y las neurociencias. Universidad de Zulia, Repositorio Acádemico ISSN 1012-1587, 1-17.

Buchelli, A. (2008). Comportamiento e influencia sociales . España.

Erizagaba, R. (2013). Comunicacion versus Publicidad externa . Pensar, 215.

Fischer, L., \& Espejo, J. (2013). Promonegocios.Net. Recuperado el 2017, de Publicidad engañosa: https:/www.promonegocios.net/publicidad/publicidad-enganosa.html

Guinn, O., Allen, \& Semenik. (2010). Publicidad. Mexico: Thomson Editores.

Hugalde, E. (2013). Consecuencias de una mala alimentacion. Mexico: http://www.vix.com/es/imj/salud/2010/10/25/consecuencias-de-una-mala-alimentacion.

Kaufer-Horwitz, M., Pérez-Lizaur, A., \& Arroyo, P. (2015). Nutriología Médica. Madrid: Medica Panamericana.

Ochoa, F., \& Ojeda, S. (2013). Mala alimentacion en los estudiantes. Dominio de las Ciencias .

Osorio, J. (2012). Desarrollo de la conducta alimentaria en la juventud . Scielo, Revista Chile Nutricional.

Rios, M. S., \& Mateos, J. (2009). Nutricion y Alimentación: Nuevas perspectivas. Mexico : McGraw Hill.

Rosa, L. (2010). La publicidad: una estrategia de comunicación . Dominio de las Ciencias .

Salas, L. G. (2011). Eduacación alimentaria: Manual indispensable sobre el comportamiento saludable. Madrid: Trillas.

Thompson, I. (2005). Definición de Publicidad. Madrid: American Marketing Asociation.

Todo Marketing. (10 de 2014). Marketing y Nutrición. Recuperado el 2017, de http://www.todomktblog.com/2014/01/publicidad-nutricion.html 